\title{
Cytology of Endolymphatic Sac Tumor
}

Bettina A. Murphy, M.D., Kim R. Geisinger, M.D., Simon Bergman, M.D.

Department of Pathology, Wake Forest University Baptist Medical Center, Winston-Salem, North Carolina

A 77-year-old man presented with decreased mental status and an enhancing partially cystic tumor along the left tentorium on magnetic resonance imaging after mastoidectomy and petrosectomy for an "auditory canal tumor." Smears of the aspirated cyst fluid revealed rare epithelial cell clusters, some with papillary features, foamy macrophages, and blood. The cells were orderly, with fairly bland nuclei and well-defined cell borders. The cell block contained similar epithelium, with cells containing eosinophilic and focally vacuolated cytoplasm, some with pigmented granules resembling hemosiderin. Numerous foam cells were also present. Review of the patient's previous and concurrent resection material showed an endolymphatic sac tumor, a rare neoplasm that arises in the endolymphatic sac in the temporal bone. The previously undescribed cytologic features of this rare neoplasm are discussed.

KEY WORDS: Cytology, Endolymphatic sac tumor, Inner ear neoplasms.

Mod Pathol 2001;14(9):920-924

Endolymphatic sac tumors (ELST) are extremely rare neoplasms that arise in the endolymphatic sac in the posterior-medial petrous portion of the temporal bone. Although the clinicopathologic and radiographic features have been well documented, the cytologic features of ELST have not been previously described (1-5). We report the cytologic findings of an ELST from the cyst fluid of a recurrent ELST in the left temporal region of a 77 -year-old man.

\section{CASE REPORT}

A 77-year-old white man presented in the fall of 1999 with decreased mental status and magnetic

Copyright (C) 2001 by The United States and Canadian Academy of Pathology, Inc.

VOL. 14, NO. 9, P. 920, 2001 Printed in the U.S.A.

Date of acceptance: April 11, 2001.

Address reprint requests to: Simon Bergman, M.D., Wake Forest University/Baptist Medical Center, Department of Pathology, Medical Center Blvd., Winston-Salem, NC 27157-1072; e-mail: sberman@wfubmc.edu fax: 336-716-7595. resonance imaging results demonstrating an enhancing tumor mass along the left tentorium with multiple adjacent cysts, the largest of which was approximately $4.3 \mathrm{~cm} \times 3.8 \mathrm{~cm}$. A midline shift was present. The patient had a history of left mastoidectomy performed at another institution for an "auditory canal" tumor originally diagnosed in the 1970s. In 1989, the tumor recurred, and he underwent left petrosectomy, also at another institution where the diagnosis was a ceruminous gland tumor. A consultant's opinion of that material was that it represented an adenocarcinoma of endolymphatic sac origin. For the patient's most recent surgery, he was taken to the operating room for left temporal-parietal craniotomy for tumor debulking and cyst drainage. The cyst fluid was described by the surgeon as yellow tinged. The patient's surgery was without complications, and he was subsequently released from the hospital.

\section{MATERIALS AND METHODS}

The cyst fluid was processed by a concentration cytocentrifuge technique, and smears were Papanicolaou stained. The cell block was formalin fixed, paraffin embedded, and stained with hematoxylin and eosin. Immunostains performed on the surgical resection material using an automated system (Ventana, Tucson, AZ) were as follows: cytokeratin AE1/AE3 in a 1:200 dilution, with pepsin digestion, S-100 protein in a 1:10,000 dilution with pepsin digestion, and glial fibrillary acid protein (GFAP) in a 1:300 dilution. The immunostains were manufactured by DAKO (Carpenteria, CA). Additional immunostains performed on the resection material were $\alpha$-fetoprotein, chromogranin, desmin, epithelial membrane antigen, human chorionic gonadotropin, muscle-specific actin, neuron-specific enolase, placental alkaline phosphatase, and vimentin (Table 1). Additionally, stains for estrogen receptors and progestin receptors were performed manually. These antibodies were manufactured by Ventana.

The smears of the cyst fluid revealed epithelial cell clusters, some with papillary features (Figs. 1 and 2). The cells had small- to medium-sized nuclei arranged in an orderly pattern. The nuclei were 
TABLE 1. Antibody Panel

\begin{tabular}{lrl}
\hline \multicolumn{1}{c}{ Substance } & Dilution & \multicolumn{1}{c}{ Supplier } \\
\hline$\alpha$-fetoprotein & $1: 400$ & DAKO, Carpenteria, CA \\
Chromogranin & $1: 100$ & DAKO, Carpenteria, CA \\
Cytokeratin AE1/AE3 & $1: 200$ & DAKO, Carpenteria, CA \\
Desmin & $1: 50$ & DAKO, Carpenteria, CA \\
Epithelial membrane antigen & $1: 200$ & DAKO, Carpenteria, CA \\
Glial fibrillary acidic protein & $1: 300$ & DAKO, Carpenteria, CA \\
Human chorionic gonadotropin & $1: 500$ & DAKO, Carpenteria, CA \\
Muscle-specific actin & $1: 50$ & Enzo, Farmington, NY \\
Neuron-specific enolase & $1: 50$ & DAKO, Carpenteria, CA \\
Placental alkaline phosphatase & $1: 20$ & Biomedia, CA \\
S-100 protein & $1: 10,000$ & DAKO, Carpenteria, CA \\
Vimentin & $1: 3000$ & Bioenex, San Ramon, CA \\
\hline
\end{tabular}

round to oval with delicate membranes and small to inconspicuous nucleoli. Occasional nuclei were larger, evenly spaced with pale chromatin and prominent nucleoli. Cell borders were well defined and cells contained a small to moderate amount of wispy cytoplasm (Fig. 3). The background consisted of scattered foamy macrophages, a few neutrophils, and blood. Cells resembling macrophages but without distinctly foamy cytoplasms were also present and may have represented epithelial cells or macrophages. Mitotic figures were not identified.

The cell block contained numerous foam cells, some of which had small yellow-brown cytoplasmic granules resembling hemosiderin. Scattered aggregates and strips of epithelium composed of a single layer of small cuboidal to columnar cells with solitary centrally or eccentrically placed monomorphic nuclei and eosinophilic to vacuolated cytoplasms were identified (Fig. 4). Yellow-brown pigment granules were present in the cytoplasm of some of the epithelial cells. Overall, the material mimicked that of benign choroidal epithelium originating from normal tissue or a benign choroid papilloma.

The concurrent surgical resection specimen showed papillary structures and variably sized cysts lined by cuboidal, columnar, and flattened cells (Fig. 5). Small nests of epithelial cells were also present within the stroma. The cytoplasm, which sometimes contained pigment granules or numerous vacuoles, was eosinophilic. Overall, the nuclei maintained a bland appearance, although occasional enlarged nuclei with prominent nucleoli were identified. Pigment-laden macrophages were present in some of the cysts. Mitotic figures were not identified. The stroma was quite vascular in some areas but in elsewhere was dense and sclerotic. Focally, the stroma immediately beneath the epithelium contained a slightly pleomorphic population of stromal cells with nuclei larger than those of the epithelial cells. These underlying cells did not stain positively for cytokeratin, whereas the surface epithelial cells were cytokeratin positive. All cells were negative for S-100 protein and GFAP. Estrogen and progesterone receptors were also negative. Ad- ditionally, immunostains for $\alpha$-fetoprotein, chromogranin, desmin, epithelial membrane antigen, human chorionic gonadotropin, muscle-specific actin, neuron-specific enolase, placental alkaline phosphatase, and vimentin were negative. The cells also stained focally weakly with periodic acid-Schiff after diastase digestion. Review of the patient's previous surgical material (1989) showed similar histologic findings (Fig. 6).

\section{DISCUSSION}

ELST are rare neoplasms that arise in the temporal bones. They are slightly more common in females than males and occur from the 1st to 8th decades of life, with the median age of diagnosis in the 4 th decade (1). For years, the terminology and origin of this tumor were in question. In the literature, many different names have been attributed to neoplasms; based on their clinical, radiological, and pathological descriptions, these names appear to represent ELST. These appellations include primary adenoma and adenocarcinoma of the temporal bone, adenomatous tumors of the middle ear and mastoid (papillary type), and aggressive papillary middle-ear tumor (6-11). Some publications describing this neoplasm have observed that tumors that occurred in the region of the temporal bone, had a papillary pattern, and pursued an aggressive clinical course with multiple local recurrences, despite their bland microscopic appearance (10-12).

In 1989, Heffner (2) published a series of 20 cases of these temporal bone lesions and suggested their origin in the endolymphatic sac of the petrous bone. In this article, he proposed the term lowgrade adenocarcinomas of probable endolymphatic sac origin. The patients in this series presented with variable degrees of unilateral hearing loss. Additional symptoms reported include tinnitus, facial nerve paresis, and vertigo (3-5). Although most cases appear sporadically, cases of ELST have also been known to occur in patients with Von HippleLindau syndrome $(1,17)$. Radiographically, bone destruction in the area of the posterior medial aspect of the temporal bone is common.

Histologically, these are papillary and cystic neoplasms with fronds lined by a single layer of cuboidal to columnar cells and spaces lined by cuboidal to flattened cells. The cells have bland nuclei, which tend to be basally oriented, and cytoplasmic vacuolation due to glycogen is common. The stroma ranges from cellular to hyalinized and is frequently well vascularized. Areas of recent and remote hemorrhage with hemosiderin deposits and cholesterol clefts may be present. The cystic areas have been described as containing periodic acid-Schiff-positive proteinaceous material, which mimics thyroid 

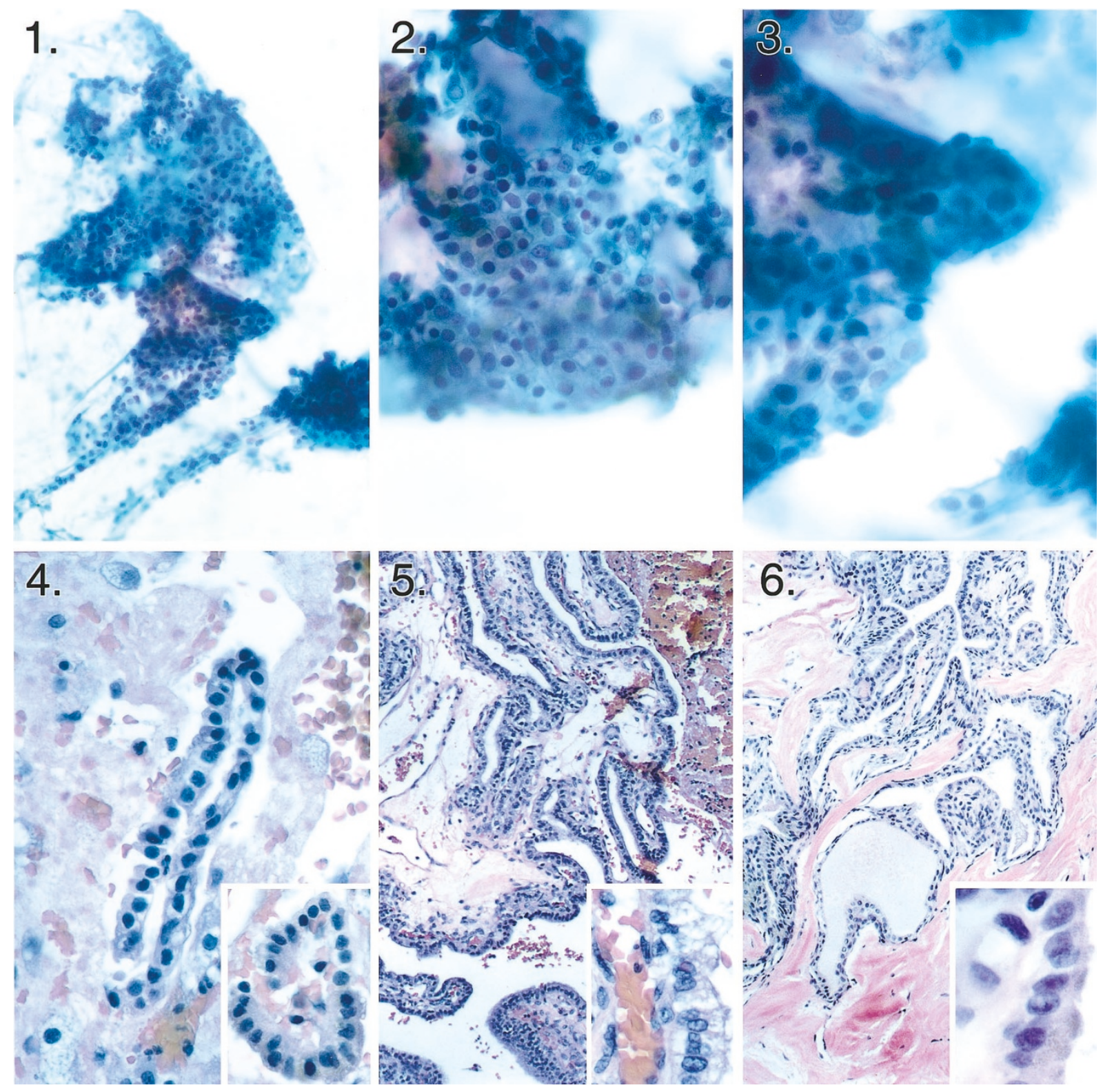

FIGURE 1. FNA of cyst fluid with papillary epithelial clusters characterized by bland nuclei and well-defined cell borders (10×).

FIGURE 2. FNA of cyst fluid with papillary epithelial clusters characterized by bland nuclei and well-defined cell borders $(20 \times)$.

FIGURE 3. FNA of cyst fluid with papillary epithelial clusters characterized by bland nuclei and well-defined cell borders (40×).

FIGURE 4. Cell block from cyst fluid showing strips of cuboidal epithelial cells characterized by bland nuclear features. (hematoxylin and eosin, $40 \times$; inset, $40 \times$ ).

FIGURE 5. Concurrent surgical resection specimen showing endolymphatic sac tumor containing papillary structures and cysts lined by epithelial cells characterized by bland nuclei. (hematoxylin and eosin, $20 \times$; inset, $40 \times$ ).

FIGURE 6. Previous surgical resection of endolymphatic sac tumor with similar histology to current tumor in Figure 5. (hematoxylin and eosin, $20 \times$; inset, $40 \times)$.

colloid. In fact, these tumors may be mistaken for metastatic papillary thyroid cancer (5). Immunohistochemically, these tumors are positive for cytokeratin with a minority displaying positivity for S-100 protein, GFAP, synaptophysin, and Leu 7. ELST behave as low-grade carcinomas: they may destroy bone and brain parenchyma and recur locally but have not been shown to metastasize.

To our knowledge, the cytomorphology of these tumors in fine-needle aspiration biopsy or in fluids has never been described. Given the overall bland nuclear features of the epithelium in the smears and lack of a true papillary pattern on the cell block, it would be difficult if not impossible to diagnose this as an ELST or even as a malignant neoplasm based purely on the cytomorphology. In fact, our initial impression was that of choroid plexus epithelium because no site or clinical information other than "brain cyst" was provided to us. Upon retrieval of the patient's history and prior material and review of the concurrent histopathology, it became clear that the material in the smears and cell 
block represented an ELST. Thus, knowledge of the patient's clinical history and radiographic findings are crucial in arriving at the diagnosis of an ELST or even suggesting this possibility based on cytology alone.

Entities in the differential diagnosis include normal choroid plexus epithelium, choroid plexus neoplasms, papillary ependymoma, and metastatic tumors such as papillary carcinoma of the thyroid and renal cell carcinoma. Choroid plexus neoplasms comprise $<1 \%$ of intracranial tumors and usually occur within the lateral ventricles of children and within the fourth ventricle of adults (13). Most commonly they present in the 1st decade of life with hydrocephalus. Normal choroid plexus epithelium has a cobblestone appearance that was not apparent in our case (14). Choroid plexus papillomas (CPP) consist of a layer of cuboidal to columnar epithelium present on delicate fibrovascular fronds. Their epithelium is more crowded and contains cells with slightly increased nuclear to cytoplasmic ratios compared to normal choroid plexus. Papillomas contain cells ranging from those with bland cellular features to those with significant atypia and increased mitotic figures (atypical papillomas). Cytologic smears show papillae lined by cuboidal to columnar cells with granular to vacuolated cytoplasms. Single and small clusters of epithelial cells are also present (15). Overall, the cells may appear identical to those seen in normal choroid plexus epithelium, which are often single and round to elongate in fluids. Thus, the size of the lesion (to differentiate normal from neoplastic choroid plexus) and clinical and radiographic presentation are important. Choroid plexus carcinomas show unequivocally malignant features with a complex architecture, striking nuclear atypia, and numerous mitotic figures $(13,14,16)$. Immunohistochemically, papillomas are positive for cytokeratin and diffusely positive for S-100 protein. GFAP may also be positive. The carcinomas are less likely to show strong positivity for S-100 protein (13). One could differentiate choroid plexus carcinomas from ELST by the anatomic location and cytologic features. Distinguishing ELST from choroid plexus papilloma or normal choroid plexus on cytomorphology alone could be difficult as all contain bland epithelium, which may closely resemble one another. Hemosiderin has been seen in cells of normal choroid plexus so this may not be a useful differentiating feature (16). Cytoplasmic vacuolization may be seen in CPP; however, a positive periodic acid-Schiff stain after diastase digestion would support ELST. Strong S-100 positivity would favor a choroid plexus origin.

Intracranial ependymomas are most common in children but also occur in adults, presenting with increased cranial pressure or mass effect. These neoplasms are intraventricular or periventricular and frequently calcified (14). It may be impossible to distinguish these from CPP with cytomorphology alone (15). The individual cells of ependymomas resemble normal ependymal cells with their cuboidal to columnar contours, bland nuclei, moderate amounts of lacy cytoplasm, and well-defined cell borders. In fluids, the presence of small clusters favors ependymoma over normal ependymal cells. Rosettes are commonly seen in fine needle aspiration biopsy (FNAB) of ependymomas but are infrequent in CSF (16). On the basis of these features, it may also be impossible to distinguish ELST from ependymoma on cytomorphology alone. Again, location and clinical presentation would be necessary for a more specific diagnosis.

Metastatic carcinomas are also in the differential diagnosis as ELST have been mistaken for metastatic papillary thyroid carcinoma and renal cell carcinoma $(2,4)$. FNAB smears of papillary thyroid carcinoma are frequently cellular and show crowded cohesive aggregates of epithelial cells with enlarged oval nuclei. Marginated nucleoli, nuclear grooves, nuclear pseudoinclusions, and fine nuclear chromatin are important diagnostic criteria (16). Smears of ELST would be expected to be hypocellular, with orderly epithelial groups consisting of cells without nuclear grooves or pseudoinclusions. Additionally, papillary carcinomas will show staining for thyroglobulin and thyroid transcription factor-1, as well as cytokeratin positivity.

As with clear cell renal carcinoma, cytoplasmic vacuolization is present in some of the tumor cells of ELST. One would expect hypercellular FNAB smears consisting of cohesive aggregates and single cells with cytoplasmic clearing. The nuclei in some renal cell carcinomas may be relatively bland and thus impossible to distinguish as carcinoma on FNAB. In other cases, marked nuclear pleomorphism and prominent nucleoli would enable a diagnosis of carcinoma (2). Clinical history is also important.

ELST are very uncommon neoplasms for which the histogenesis has only recently been defined. The histologic findings in combination with the clinical and radiographic presentation allow for an accurate diagnosis. The cytologic differential diagnosis is a short one; however, the accurate diagnosis of ELST based solely on cytomorphology is a difficult one. In combination with the clinical and radiographic findings, this diagnosis may be suggested or strongly favored.

\section{REFERENCES}

1. Weing B, Hefner D. Endolymphatic sac tumors: fact or fiction. Adv Anat Pathol 1996;3:378-87.

2. Heffner DK. Low-grade adenocarcinoma of probable endolymphatic sac origin: a clinicopathologic study of 20 cases. Cancer 1989;64:2292-302. 
3. Reijneveld J, Hanlo R, Groenewoud G, Jansen G, van Overbeeke K, Tulleken C. Endolymphatic sac tumor: a case report and review of the literature. Surg Neurol 1997;48:368-73.

4. Kempermann G, Neumann H, Volk B. Endolymphatic sac tumours. Histopathology 1988;33:2-10.

5. Stendel R, Suess O, Prosenc N, Funk T, Brock M. Neoplasm of endolymphatic sac origin: clinical, radiological and pathological features. Acta Neurochir (Wien) 1998;140:1083-7.

6. Batsakis J, el-Naggar AK. Papillary neoplasms (Heffner's tumors) of the endolymphatic sac. Ann Otol Rhinol Laryngol 1993;102:648-51.

7. Glasscock M, McKennan K, Levine S, Jackson G. Primary adenocarcinoma of the middle ear and temporal bone. Arch Otolaryngol Head Neck Surg 1987;113:822-4.

8. Eby T, Makek M, Fisch U. Adenomas of the temporal bone. Ann Otol Rhinol Laryngol 1988;97:605-12.

9. Benecke JE, Noel FL, Carberry JN, House JW, Patterson M. Adenomatous tumors of the middle ear and mastoid. Am J Otol 1990;11:20-6.

10. Gaffey M, Mills S, Fechner R. Aggressive papillary middle-ear tumor: a clinicopathologic entity distinct from middle-ear adenoma. Am J Surg Pathol 1988;12:790-7.
11. Poe D, Tarlov E, Thomas C, Kveton J. Aggressive papillary tumors of temporal bone. Otolaryngol Head Neck Surg 1993; 108:80-6.

12. Mills S, Fechner R. Middle ear adenoma: a cytologically uniform neoplasm displaying a variety of architectural patterns. Am J Surg Pathol 1984;8:677-85.

13. Coffin C, Wick M, Braun J, Dehner L. Choroid plexus neoplasms: clinicopathologic and immunohistochemical studies. Am J Surg Pathol 1986;10:394-404.

14. Burger PC, Scheithauer BW. Atlas of tumor pathology: tumors of the central nervous system. Washington, D.C.: Armed Forces Institute of Pathology; 1994.

15. Buchino J, Mason K. Choroid plexus papilloma: report of a case with cytologic differential diagnosis. Acta Cytol 1992;36: 95-97.

16. DeMay RM. The art and science of cytopathology: exfoliative cytology. Chicago: ASCP Press; 1996. p. 434, 441-442, 1186.

17. Kempermann G, Neumann HP, Scheremet R, Volk B, Mann W, Gilsbach J, et al. Deafness due to bilateral endolymphatic sac tumors in a case of von Hippel-Lindau syndrome. J Neurol Neurosurg Psych 1996;61:318-320. 